\title{
Role of dynamical modes in changing southern hemisphere climate
}

\author{
Jorgen S. Frederiksen ${ }^{1} \quad$ Carsten S. Frederiksen ${ }^{2}$
}

(Received 19 January 2011; revised 19 April 2011)

\begin{abstract}
We examine the ability of leading dynamical eigenmodes to capture important properties of the changing southern hemisphere weather systems during the 20th century. A global atmospheric model uses three dimensional July basic states taken from observations for the periods 1949-1968, 1975-1994 and 1997-2006. Iterative eigensolvers using the Arnoldi method of Krylov subspace reduction are contrasted with direct methods of matrix construction and solution. The results are related to the large changes in winter weather systems, rainfall and circulation that have occurred during the last sixty years in the Australian region. We find that storm track and onset-of-blocking modes growing on the subtropical jet have significantly reduced growth rates since the 1970s. On the other hand, there has been significant increase in the growth rates of northwest cloud-band modes and intraseasonal oscillations that cross Australia, and some smaller increase in the intensity of African easterly waves.
\end{abstract}

http://anziamj.austms.org.au/ojs/index.php/ANZIAMJ/article/view/3892 gives this article, (c) Austral. Mathematical Soc. 2011. Published May 4, 2011. ISSN 1446-8735. (Print two pages per sheet of paper.) Copies of this article must not be made otherwise available on the internet; instead link directly to this URL for this article. 


\section{Contents}

1 Introduction

C73

2 Primitive equation model

C74

3 Eigenvalue-eigenvector system

4 Basic states

5 Southern hemisphere dynamical modes $\quad$ C79

5.1 Storm track modes . . . . . . . . . . . . . . C79

5.2 Mid-latitude blocking modes . . . . . . . . . . . . . C C81

5.3 Northwest cloudband modes . . . . . . . . . . . . C83

5.4 Intra-seasonal oscillation modes . . . . . . . . . . C85

5.5 Other important dynamical modes . . . . . . . . . C85

6 Conclusion

C86

References

C87

\section{Introduction}

Leading eigenmodes of the linearised atmospheric equations of motion have been widely used to understand the dynamics of weather systems [1] and their predictability [2]. Of course, nonlinear effects cause modifications of the disturbances as they amplify [3]. Nevertheless, normal modes of climatological basic states [4] and finite time, normal modes of time evolving basic states [2, 5] capture many of the important properties of atmospheric disturbances and their predictability.

Recently, we applied normal mode theory to understand the likely causes of the dramatic reduction in winter rainfall that occurred over southern 
Australia and particularly over the south west of Western Australia since the 1970s [6]. We found that the rainfall reduction was related to systematic changes in the July southern hemisphere circulation, with a weakening of the subtropical jetstream and reduction in the growth rates of storm track modes crossing southern Australia. We reviewed observational studies linking rainfall intensity and growth rates of storms. That study compared the time intervals 1949-68 and 1975-94.

Here we firstly outline numerical and computational techniques for solving large eigenproblems. Then we consider applications for understanding the causes of the changes in weather systems and rainfall during the the more recent time interval 1997-2006 when southeastern Australia experienced a severe drought. We again study the properties of July extra-tropical storm track modes but as well, for all three periods, we consider a wider range of dynamical modes affecting different parts of Australia. A particular aim is to see whether the changes in these leading dynamical modes are broadly consistent with the trends in July rainfall over the period 1950-99 shown in figure 1.

Section 2 details the primitive equations describing atmospheric thermodynamics. Section 3 describes methods of linearising the equations and obtaining the leading eigenmodes. Section 4 describes basic states for the three time intervals in July. Section 5 details properties of the dynamically important leading eigenmodes associated with changes in Australian weather systems and rainfall. Section 6 concludes.

\section{Primitive equation model}

Our study of the changes in dynamical instability modes during the 20th century employs a two level primitive equation model of atmospheric motions on the spherical earth, including cumulus convection and evaporation [6]. 


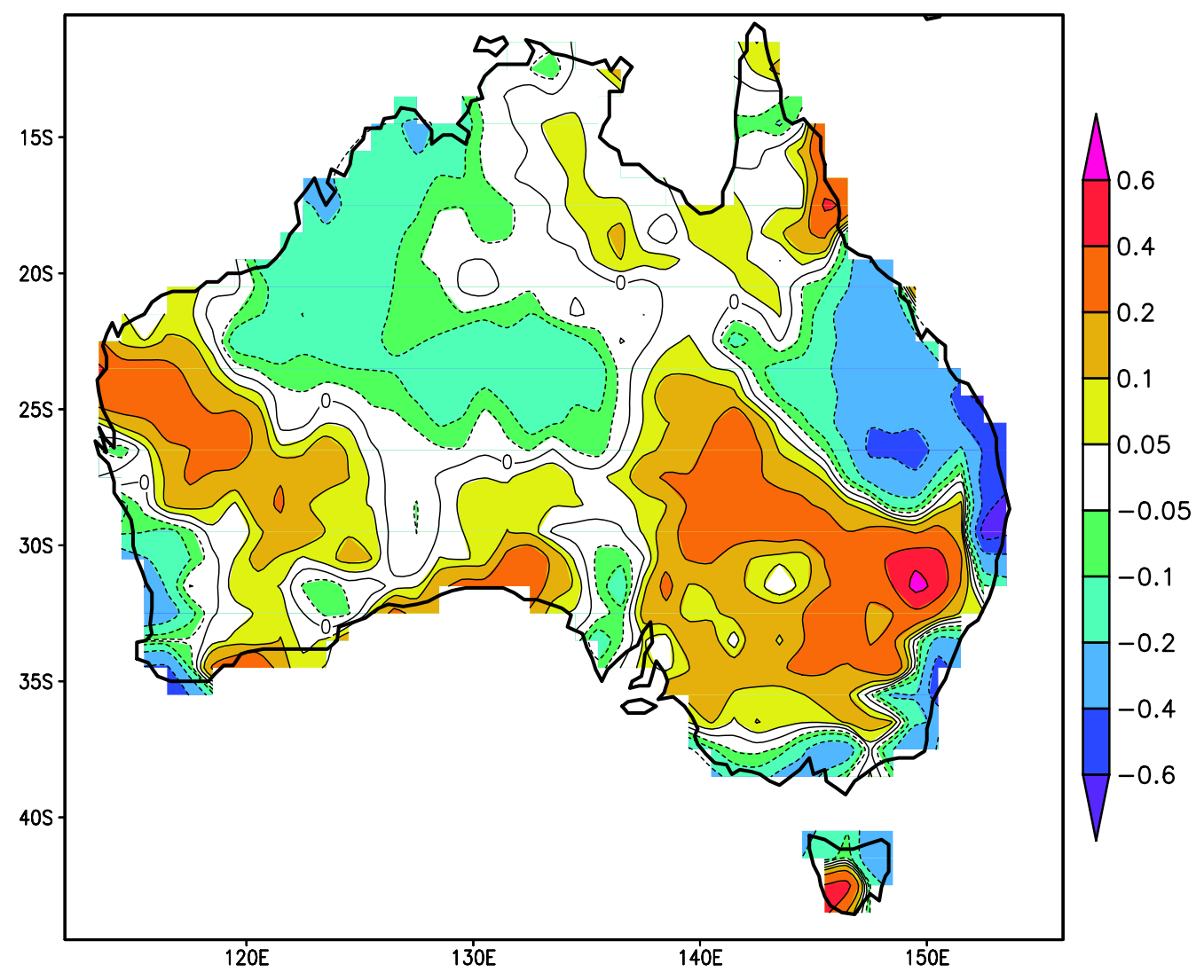

Figure 1: Trends in Australian July rainfall (mm/month per year). 
These equations take the form

$$
\begin{aligned}
& \frac{\partial \nabla^{2} \psi}{\partial \mathrm{t}}=-\mathrm{J}\left(\psi, \nabla^{2} \psi+2 \Omega \mu+\mathrm{h} / 2\right)-\mathrm{J}\left(\tau, \nabla^{2} \tau-\mathrm{h} / 2\right)+\mathrm{J}\left(\chi, \nabla^{2} \chi\right) \\
& +\nabla \cdot\left(\left(\nabla^{2} \tau-\mathrm{h} / 2\right) \nabla \chi\right)+\nabla \cdot\left(\nabla^{2} \chi \nabla \tau\right) \\
& -\mathrm{K} \nabla^{2}(\psi-\tau)-\mathrm{K}^{\prime} \nabla^{6} \psi+\mathrm{F}_{\psi}, \\
& \frac{\partial \nabla^{2} \tau}{\partial \mathrm{t}}=-\mathrm{J}\left(\tau, \nabla^{2} \psi+2 \Omega \mu+\mathrm{h} / 2\right)-\mathrm{J}\left(\psi, \nabla^{2} \tau-\mathrm{h} / 2\right) \\
& +\nabla \cdot\left(\left(\nabla^{2} \psi+2 \Omega \mu+\mathrm{h} / 2\right) \nabla \chi\right)-K \nabla^{2}(\tau-\psi) \\
& -\mathrm{K}^{\prime} \nabla^{6} \tau+\mathrm{F}_{\tau} \text {, } \\
& \frac{\partial \nabla^{2} \chi}{\partial \mathrm{t}}=-\mathrm{J}\left(\chi, \nabla^{2} \psi+2 \Omega \mu+\mathrm{h} / 2\right)+\nabla \cdot\left(\left(\nabla^{2} \psi+2 \mu+\mathrm{h} / 2\right) \nabla \tau\right) \\
& +\nabla \cdot\left(\left(\nabla^{2} \tau-\mathrm{h} / 2\right) \nabla \psi\right)+\nabla^{2}(\nabla \psi \nabla \tau-\mathrm{J}(\psi, \chi)+\theta) \\
& -\mathrm{K} \nabla^{2} \mathrm{x}-\mathrm{K}^{\prime} \nabla^{6} \mathrm{x}+\mathrm{F}_{\mathrm{x}} \\
& \frac{\partial \theta}{\partial \mathrm{t}}=-\mathrm{J}(\psi, \theta)-\mathrm{J}(\tau, \sigma)+\nabla \cdot \sigma \nabla \chi-\mathrm{K}^{\prime} \nabla^{4} \theta+\mathrm{F}_{\theta}, \\
& \frac{\partial \sigma}{\partial t}=-\mathrm{J}(\psi, \sigma)-\mathrm{J}(\tau, \theta)+\nabla \cdot \theta \nabla \chi-\mathrm{K}^{\prime} \nabla^{4} \sigma+\mathrm{F}_{\sigma} .
\end{aligned}
$$

Here, the Jacobian

$$
J(X, Y)=a^{-2}\left(\frac{\partial X}{\partial \lambda} \frac{\partial Y}{\partial \mu}-\frac{\partial X}{\partial \mu} \frac{\partial Y}{\partial \lambda}\right)
$$

where $\lambda$ is the longitude, $\mu$ is the latitude and $t$ is the time. Also $\psi$ is the average streamfunction between the upper $(300 \mathrm{hPa})$ and lower $(700 \mathrm{hPa})$ pressure levels, $\tau$, the vertical shear streamfunction, is half the difference between the upper and lower level streamfunctions, $\chi$ is the lower level velocity potential (equal to minus the upper level velocity potential), and $\theta$ and $\sigma$ are the mean and vertical shear potential temperatures respectively. Here, $a$ is the radius of the earth, $\Omega$ is the earth's angular velocity, $h$ is the topography, $\mathrm{K}$ and $\mathrm{K}^{\prime}$ are drag and bi-harmonic diffusion coefficients and $\mathrm{F}$ denotes forcing functions that appear in the various equations and include cumulus convection 
and evaporation in the potential temperature equations. We use the standard parameters described in an earlier article [6, section 3].

\section{Eigenvalue-eigenvector system}

We suppose that each dynamical field is represented by a basic state and a small perturbation so that the perturbation equations are effectively linearised. We expand each of the perturbation fields and basic state fields in terms of spherical harmonics with the perturbations also having a time dependence $\exp (-i \omega t)$. Here $\omega=\omega_{r}+i \omega_{i}$ is the complex angular frequency with $\omega_{\mathrm{r}}$ being the frequency and $\omega_{i}$ the growth rate. This then results in a system of eigenvalue-eigenvector equations of the form

$$
-i \omega x=\Lambda x=M x
$$

where $\Lambda=-i \omega, x$ is the column vector of spherical harmonic spectral coefficients of the five perturbation field variables and $M$ is a matrix that depends on the basic state fields.

Formulating and solving eigenvalue systems may be challenging for large sets of complex equations. We cross checked our methodologies and eigensolvers by employing four different approaches. The first method is to construct the matrix $M$ explicitly in spectral space [1]. A second method is to linearise the equations (1)-(5), and then obtain the matrix, a column at a time, from the one step tendency resulting from initialising the equations with a particular real or imaginary part equal to unity and all other spectral components set to zero [7]. A third method is to use the second method without linearising the equations explicitly. In the third method the basic state is perturbed by a small $\epsilon$ in the real or imaginary part of a specific spectral component, with all other components unperturbed, and the matrix $M$ is obtained, a column at a time, from $\epsilon^{-1}$ times the tendency.

A fourth approach has been not to construct the full matrix $M$ explicitly but to use iterative eigensolvers following the method of Arnoldi [8]. It 
employs Krylov subspace reduction and is an efficient method for solving large eigenvalue-eigenvector problems. This method relies on the fact that forward integration of the linearised equations from general initial conditions $\mathbf{x}(0)$ will, with time, yield a field $\mathbf{x}(\mathrm{t})$ which consists largely of a small number of the fast growing eigenmodes. The field $\mathbf{x}(t)$, from which the slower growing modes have been effectively purged, is then used to construct $k \ll n$ vectors, $\mathrm{x}_{1}=\mathrm{x}(\mathrm{t}), \mathrm{x}_{2}=\mathrm{Gx}_{1}, \ldots, \mathrm{x}_{\mathrm{k}}=\mathrm{G}_{\mathrm{k}-1}=\mathrm{G}^{\mathrm{k}-1} \mathrm{x}_{1}$ where $\mathrm{G}=\exp [\mathrm{M} \Delta \mathrm{t}], \Delta \mathrm{t}$ is a timestep and $M$ and $G$ are $n \times n$ matrices. An orthonormalised basis of the Krylov subspace is defined: let $\mathbf{w}_{1}=\mathbf{x}(t) /|\mathbf{x}(t)|$ be the first normalised vector whereas the remaining of the orthonormalised vectors are

$$
\begin{aligned}
& \mathbf{v}_{j+1}=G \mathbf{w}_{j}-\sum_{l}^{j}\left(\mathbf{w}_{l}^{\top} G \mathbf{w}_{j}\right) \mathbf{w}_{l}, \\
& \mathbf{w}_{j+1}=\mathbf{v}_{j+1} /\left|\mathbf{v}_{j+1}\right|, \quad j=1,2, \ldots, k .
\end{aligned}
$$

The $\mathrm{n} \times \mathrm{k}$ matrix $\mathbf{W}_{\mathrm{k}}$ with column vectors $\left\{\mathbf{w}_{1}, \mathbf{w}_{2}, \ldots, \mathbf{w}_{\mathrm{k}}\right\}$ allows the construction of the small $k \times k$ matrix $\mathbf{H}_{k}=W_{k}^{\top} G W_{k}$ with eigenvalues $\lambda_{j}$ and eigenvectors $\mathbf{y}_{\mathfrak{j}}, \mathfrak{j}=1,2, \ldots, k$. The leading eigenvalues, $\lambda_{j}$, and vectors $\mathbf{z}_{j}=W_{k} \mathbf{y}_{j}$, may constitute good approximations to the eigenvalues and eigenvectors of $G$. Of course $G$ and $M$ have the same eigenvectors while their eigenvalues are related by $\lambda_{j}=\exp \left(\Lambda_{j} \Delta t\right)$, where $\Lambda_{j}=-i \omega_{j}$. Issues of the choice of Krylov subspace dimension $k$ and convergence criteria for the eigenvectors and eigenvalues were discussed by Wei and Frederiksen [5]: a particularly robust variant of this method was developed and checked against results using Linear Algebra Package (LAPACK) routines.

Here, rhomboidal 15 truncation is used for both the perturbation and basic state fields with the zonal wave number $m=-15, \ldots, 0, \ldots, 15$ and the total wave number $n=|m|,|m|+1, \ldots,|m|+15$. This results in a matrix of size $2480 \times 2480$. For matrices this size, both the direct approach using LAPACK and the iterative Arnoldi method are practicable [5]. 


\section{Basic states}

The basic states for this study are taken from reanalysed observations as in our recent study [6] which focused on changes in July storm track modes between the time intervals 1949-68 and 1975-94 using global climate fields as basic states. Here, we examine a wide variety of other modes that play important roles in the dynamics during these times as well as examining leading eigenmodes for 1997-2006. We recall that between the time intervals 1949-68 and 1975-94 there was a very significant reduction of $17 \%$ in the peak strength of the southern hemisphere subtropical jet stream [6, figure 1]. A similar reduction occurred between 1949-68 and 1997-2006.

\section{Southern hemisphere dynamical modes}

Next we examine the structures of the main classes of southern hemisphere modes during the three periods 1949-68, 1975-94 and 1997-2006.

\subsection{Storm track modes}

The leading southern hemisphere storm track modes for the time intervals 194968, 1975-94, 1997-2006 are modes 1, 9, and 12, all have periods of 1.3 days but, respectively, growth rates of 0.423 day $^{-1}, 0.282$ day $^{-1}$, and 0.266 day $^{-1}$, and pattern correlations with mode 1 for 1949-68 of 1.0, 0.915, and 0.896. This corresponds to reductions in growth rates of $33.5 \%$ and $37.1 \%$ during $1975-94$ and 1997-2006 respectively. Here the modes are ordered by decreasing growth rate and the mode number specifies that order.

Figure 2 shows the upper level streamfunction at a particular instance and its Root Mean Square (RMS) amplitude for mode 12 for the 1997-2006 basic state. It is very similar to mode 1 for 1949-68 and mode 9 for 1975-94, as expected from their high pattern correlations. The disturbance has a wavenumber 
(a)

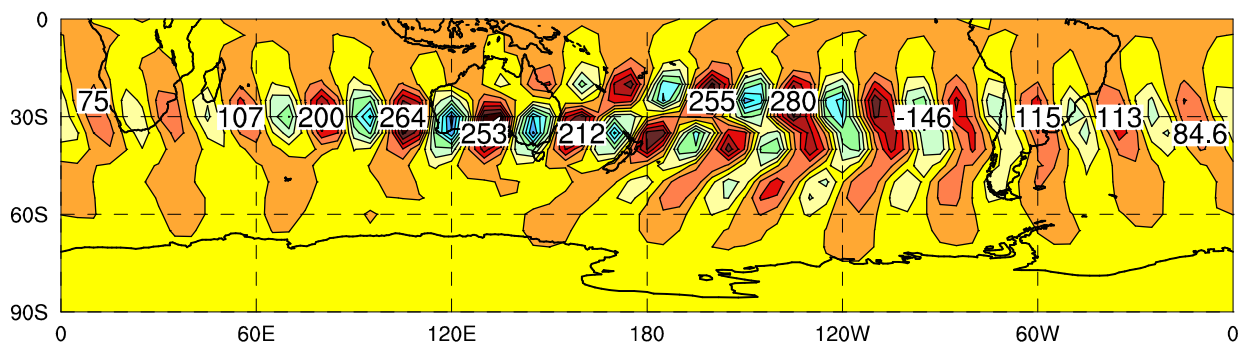

(b)

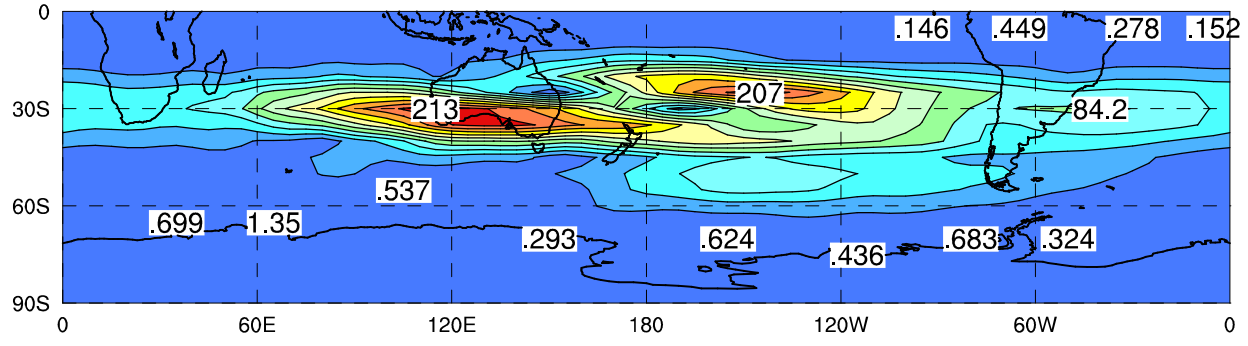

FiguRE 2: Disturbance streamfunction (and its RMS amplitude (b) at $300 \mathrm{hPa}$ for leading sH storm track mode (mode 12) for 1997-2006.

of around twelve in the regions of large amplitude over southern Australia and consists of a series of eastward propagating troughs and ridges. As the lows and highs move eastward they amplify to reach a maximum in preferred regions of large RMS amplitude. The RMS amplitude is calculated through a random phase ensemble average [1]. The mode has its largest amplitude focused over south western Australia and secondary maxima over the central Southern Pacific Ocean; the divergence field has a very similar structure. Precipitation is essentially determined by the lower level vertical velocity in pressure coordinates, and in our model this is proportional to the magnitude of the upper level divergence, for which the maximum is focused over southern Australia. Thus the significant reduction in growth rates of storm track modes in the two latter time intervals is consistent with the reduction in rainfall (figure 1) over southern Australia, and southwest Western Australia in particular, since the 1970s. Mode 12, shown in figure 2, is the fastest growing of a class of cyclogenesis modes with zonal wavenumbers between six 


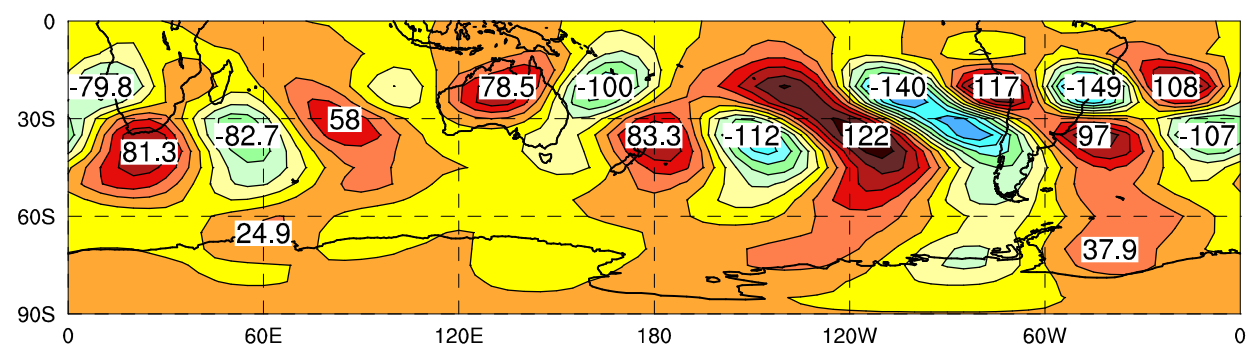

FigURE 3: Disturbance streamfunction for leading SH mid-latitude blocking mode (mode 26) at $300 \mathrm{hPa}$ for 1997-2006.

and twelve and large amplitudes in the Australian region.

\subsection{Mid-latitude blocking modes}

For the time intervals 1949-68, 1975-94, and 1997-2006 the leading southern hemisphere mid-latitude onset-of-blocking modes [1] are modes 30, 21, and 26, all have periods of six days but, respectively, growth rates of 0.257 day $^{-1}$, 0.228 day $^{-1}$, and 0.207 day $^{-1}$, and pattern correlations with mode 30 for $1949-68$ of $1.0,0.460$, and 0.640 . Thus there are reductions in growth rates of $11.2 \%$ and $19.2 \%$ in 1975-94 and 1997-2006 compared with 1949-68.

Mid-latitude, dipole, onset-of-blocking modes are eastward propagating, have longer periods than cyclogenesis modes (six days compared with 1.3 days), and are of somewhat larger scale. They have dipole structures in the latitudinal direction in the major regions of observed southern hemisphere blocking [1]. Figure 3 shows an example of a mid-latitude onset-of-blocking mode (mode 26) for the 1997-2006 basic state; the upper level streamfunction has dipole structures between Australia and New Zealand, extending downstream over the central Pacific Ocean and there are also dipole structures over the southern Atlantic ocean. As noted above, for the time intervals 1949-68 and 1975-94, there are also onset-of-blocking modes with dipole wave trains downstream 
(a)

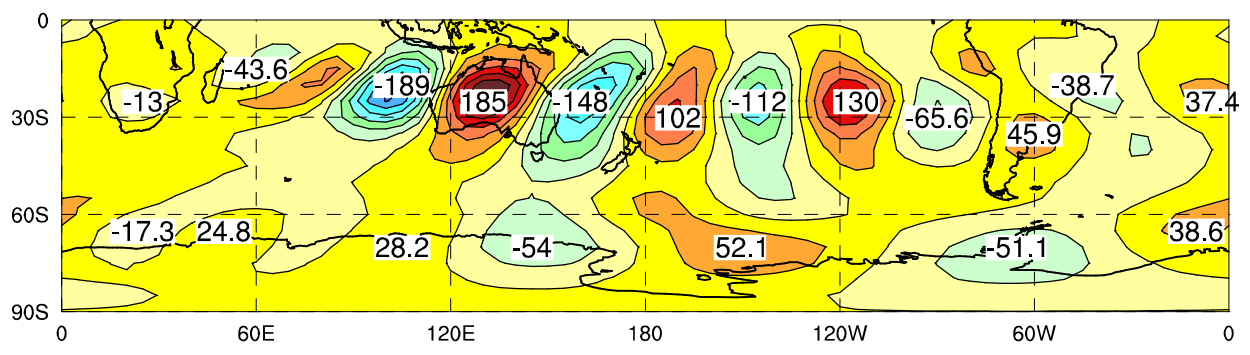

(b)

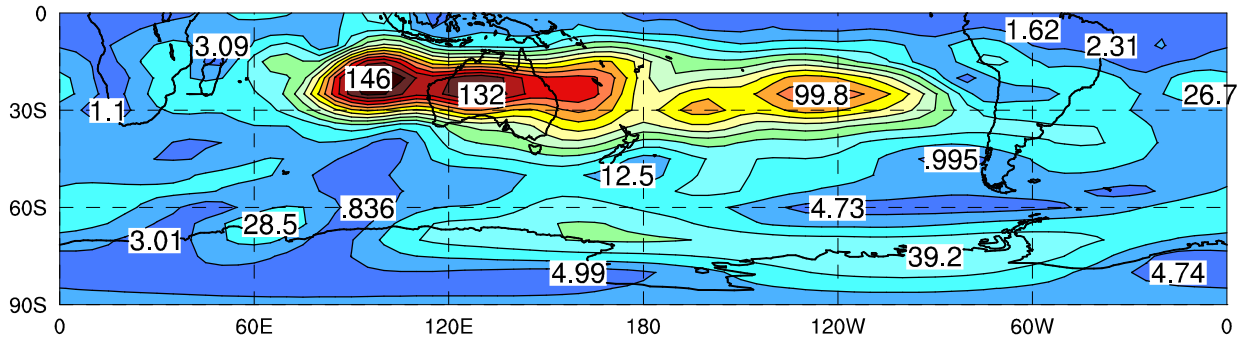

(c)

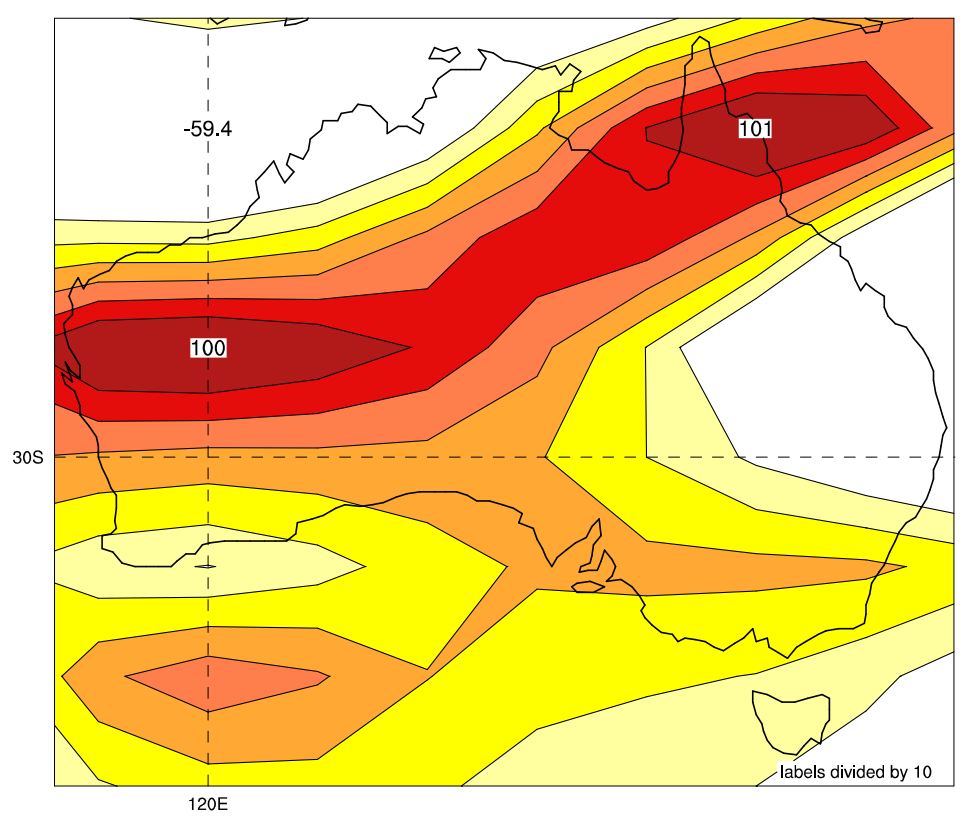

FiguRE 4: (a) disturbance streamfunction, (b) its RMS amplitude, and (c) divergence field over Australia for leading north west cloud-band mode (mode 12) at $300 \mathrm{hPa}$ for $1975-1994$. 
of Australia, as in figure 3. We expect that the growth rate reductions in 1975-94 and 1997-2006 compared with 1949-68 at least partly reflect the reductions in baroclinicity of the subtropical jet (that cause even greater reductions in the growth rate of storm track modes). The blocking highs in the region of New Zealand are also frequently associated with rain bearing eastcoast lows on the east coast of Australia. Again, the trends in rainfall over Australia during 1950-99 in figure 1, show reductions in rainfall along the east coast of Australia consistent with the smaller average growth rates of these larger scale modes since the 1970s.

\subsection{Northwest cloudband modes}

Figure 1 shows that there are regions of increased rainfall stretching southeast from northwest and central Western Australia. Here we examine the extent to which these results may be related to changes in northwest cloud band disturbances [9]. The leading northwest cloud band modes for the time intervals 1949-68, 1975-94, and 1997-2006, are modes 43, 12, and 7, all have periods of eight days but, respectively, growth rates of 0.210 day $^{-1}$, 0.262 day $^{-1}$, and 0.305 day $^{-1}$, and pattern correlations with mode 43 for 1949-68 of 1.0, 0.840, and 0.637 . This corresponds to increases in growth rates of $24.9 \%$ and $45.4 \%$ during $1975-94$ and 1997-2006 respectively.

The Northwest cloud-band (NWCB) modes are south eastward propagating with periods of eight days, although we also find slower growing NWCB modes with longer periods, as discussed in a different context by Frederiksen and Frederiksen [9]. Mode 12 for the 1975-94 basic state is a NWCB mode that emanates from the Indian Ocean region, crosses Australia and then propagates into the central Pacific and south across South America. Figure 4(a) shows the wavetrain of its $300 \mathrm{hPa}$ disturbance streamfunction, while figure 4(b) shows its RMS amplitude which has a secondary maximum over central Australia. The $300 \mathrm{hPa}$ divergence field depicted in figure 4(c) has a large amplitude over central Western Australia and northern Queensland. It again is of larger 


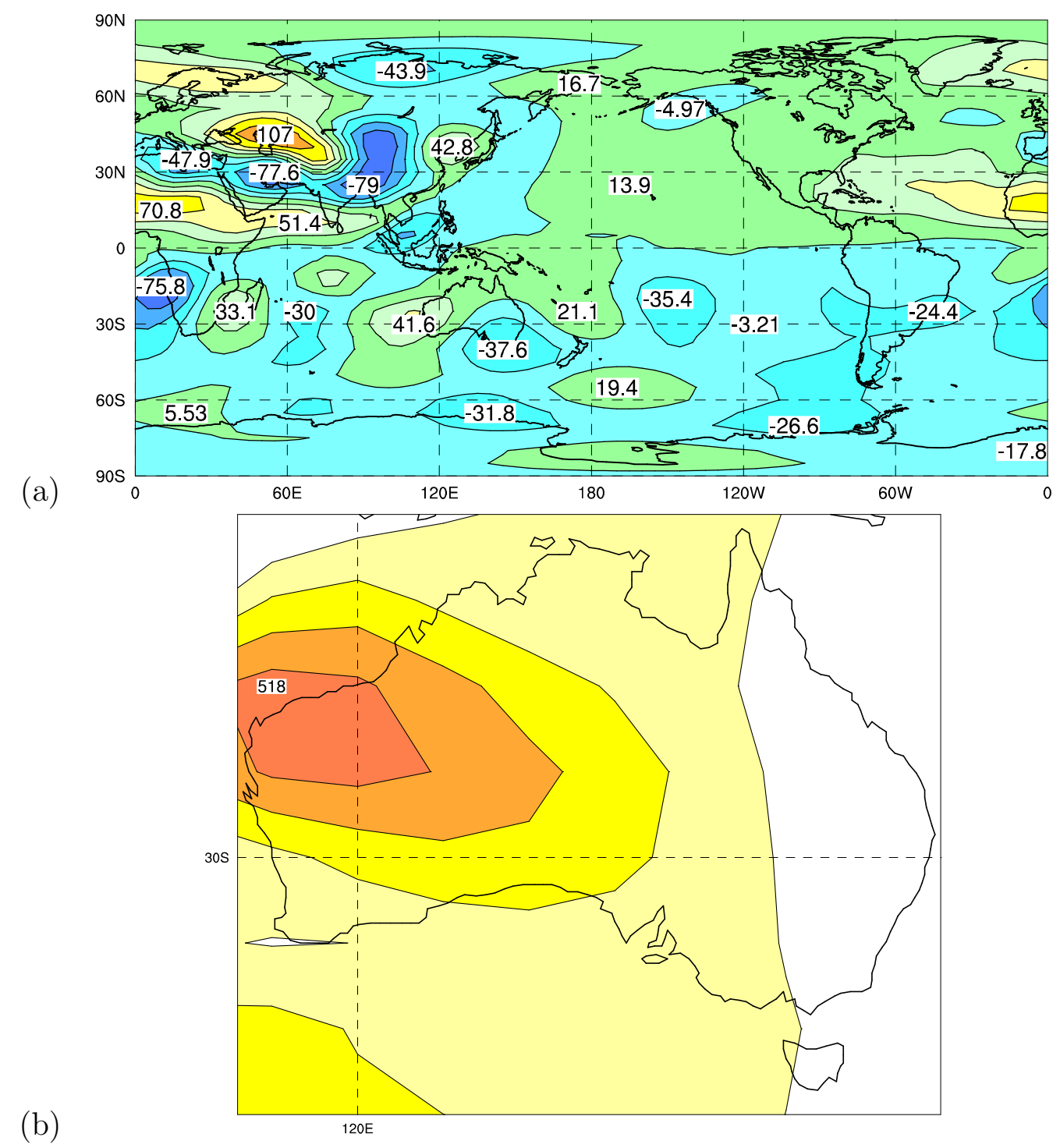

FiguRE 5: (a) global disturbance streamfunction, and (b) divergence field over Australia for leading intra-seasonal oscillation mode (mode 35 ) at $300 \mathrm{hPa}$ for $1975-1994$. 
scale than the storm track modes such as that shown in figure 2. It also differs significantly from the storm track and onset-of-blocking modes in that the NWCB growth rate for the time interval 1975-94 is $25 \%$ greater, and for 1997-2006 is 45\% greater, than for the leading NWCB for the 1949-68 basic state. Again, the increased growth rates of the NWCB modes in the later time intervals is consistent with the rainfall trends in figure 1.

\subsection{Intra-seasonal oscillation modes}

Next, we consider the leading intra-seasonal oscillation modes [4] for the time intervals 1949-68, 1975-94, and 1997-2006, they are modes 57, 35 and 33, have respective periods of 59 days, 35 days, and 25 days, growth rates of 0.144 day $^{-1}, 0.186$ day $^{-1}$, and 0.189 day $^{-1}$, and pattern correlations with mode 57 for 1949-68 of 1.0, 0.851, and 0.574. This corresponds to increases in growth rates of $28.7 \%$ and $30.6 \%$ during $1975-94$ and 1997-2006 respectively.

The July intra-seasonal oscillation modes for basic states during the second half of the 20th century have streamfunctions that extend from the equator into both hemispheres. In the southern hemisphere, eastward propagating mode 35 for the 1975-94 basic state has a wave train extending from the Indian Ocean across Australia into the central and south Pacific, as shown in figure $5(\mathrm{a})$. The $300 \mathrm{hPa}$ divergence field depicted in figure 5(b) has large amplitude over central Western Australia. Again, the structure of the flow fields and the fact that the growth rates of the intra-seasonal oscillation modes increase by about 30\% after the 1970s is consistent with the trends in the rainfall pattern in figure 1.

\subsection{Other important dynamical modes}

We find that there are groups of other leading modes that potentially play important roles in the changing climate during the second half of the 20th 
century. These modes include high latitude blocking modes, such as modes 4 and 6 for 1975-94, with significant amplitudes at high latitudes between New Zealand and Antarctica, as well as in mid-latitudes. For the three time intervals considered throughout this article, these modes have periods between five and eight days and are eastward propagating but show little systematic change in growth rates. Perhaps this is because they also grow on both the polar jet that has strengthened and on the subtropical jet that has weakened with time.

For the 1975-94 basic state, the two leading Antarctic modes are mode 3 with a period of 17 days and mode 11 with a period of 52 days. These two lower frequency modes are located principally over the Antarctic regions and consist of superpositions of zonal wavenumbers two and three. They have close similarities with the corresponding Antarctic teleconnection pattern idetified two decades ago by Frederiksen and Frederiksen [10, figure 11]. Again, there are similar teleconnection pattern modes with the basic states for 1949-68 and 1997-2006. These teleconnection patterns are little changed in growth rate or structure during the three periods of interest.

African easterly waves are westward propagating tropical waves that are generated by the African easterly jet and originate on the east coast of North Africa. They are frequent precursors of Atlantic hurricanes with about $85 \%$ of intense hurricanes having their origins as African easterly waves. The leading African easterly wave for the 1975-94 basic state is mode 7 which is a baroclinic mode (with significant vertical shear) with a period of four days and with growth rates that are between 5\% and 7\% larger post 1975 than for the earlier period of 1949-68.

\section{Conclusion}

Numerical methods and computational techniques obtain the leading eigenmodes of atmospheric primitive equations with July climatological basic 
states for 1949-68, 1975-94 and 1997-2006. The modes capture many of the important properties of the major classes of weather systems affecting the southern hemisphere. The changes in the growth rates of leading normal modes during second half of the 20th century, and the start of the 21st, are consistent with the observed trends in Australian rainfall.

\section{References}

[1] Frederiksen, J. S., The onset of blocking and cyclogenesis in southern hemisphere synoptic flows, J. Atmos. Sci., 41, 1984, 1116-1131. doi:10.1175/1520-0469(1984)041;1116:TOOBAC ¿2.0.CO;2 C73, C77, C80, C81

[2] Frederiksen, J. S., Singular vectors, finite-time normal modes and error growth during blocking, J. Atmos. Sci., 57, 2000, 312-333. doi:10.1175/1520-0469(2000)057¡0312:SVFTNM ¿2.0.CO;2 C73

[3] Frederiksen, J. S., Growth and vacillation cycles of disturbances in southern hemisphere flows, J. Atmos. Sci., 38, 1981, 1360-1375. doi:10.1175/1520-0469(1981)038;1360:GAVCOD;2.0.CO;2 C73

[4] Frederiksen, J. S., and C. S. Frederiksen, Monsoon disturbances, intraseasonal oscillations, teleconnection patterns, blocking andstorm tracks of the global atmosphere during January 1979: Linear theory, J. Atmos. Sci., 50, 1993, 1349-1372. doi:10.1175/1520-0469(1993)050;1349:MDIOTP ¿2.0.CO;2 C73, C85

[5] Wei, M., and J. S. Frederiksen, Finite-time normal mode disturbances and error growth during southern hemisphere blocking, Adv. Atmos. Sci., 22, 2005, 69-89. doi:10.1007/BF02930871 C73, C78

[6] Frederiksen, J. S. and C. S. Frederiksen, Interdecadal changes in southern hemisphere winter storm track modes, Tellus, 59A, 2007, 599-617. doi:10.1111/j.1600-0870.2007.00264.x C74, C77, C79 
[7] Frederiksen, C. S., and J. S. Frederiksen, Northern hemisphere storm tracks and teleconnection patterns in primitive equation and quasigeostrophic models. J. Atmos. Sci., 49, 1992, 1443-1458. doi:10.1175/1520-0469(1992)049;1443:NHSTAT¿2.0.CO;2 C77

[8] Arnoldi, W. E., The principle of minimized iterations in the solution of the matrix eigenvalue problem, Quart. Appl. Math., 9, 1951, 17-29. C77

[9] Frederiksen, C. S., and J. S. Frederiksen, A theoretical model of Australian Northwest cloud band disturbances and southern hemisphere storm tracks: The role of SST anomalies. J. Atmos. Sci., 53, 1996, 1410-1432. doi:10.1175/1520-0469(1996)053ز1410:ATMOAN¿2.0.CO;2 C83

[10] Frederiksen, J. S., and C. S. Frederiksen, Southern hemisphere storm tracks, blocking and low frequency anomalies in a primitive equation model, J. Atmos. Sci., 50, 1993, 3148-3168.

doi:10.1175/1520-0469(1993)050;3148:SHSTBA;2.0.CO;2 C86

\section{Author addresses}

1. Jorgen S. Frederiksen, Climate Adaptation Flagship, CSIRO Marine and Atmospheric Research, Aspendale, Victoria, Australia. mailto: Jorgen. Frederiksen@csiro. au

2. Carsten S. Frederiksen, Centre for Australian Weather and Climate Research, Bureau of Meteorology, Docklands, Victoria, Australia 